\title{
Host specificity of Asian parasitoids for potential classical biological control of Drosophila suzukii
}

\author{
Pierre Girod $^{1,2} \cdot$ Océane Lierhmann $^{1} \cdot$ Teddy Urvois $^{1} \cdot$ Ted C. J. Turlings $^{2} \cdot$ Marc Kenis $^{1} \cdot$ Tim Haye $^{1}$ (I)
}

Received: 21 February 2018 / Revised: 11 May 2018 / Accepted: 12 June 2018 / Published online: 18 June 2018

(c) The Author(s) 2018

\begin{abstract}
The Asian spotted wing drosophila, Drosophila suzukii, has recently become a serious pest of soft fruits in Europe. Classical biological control through the introduction of larval parasitoids from its native range in Asia is presently being considered. However, host specificity of potential biological control agents has to be determined to avoid releasing species that may have unintended non-target impacts. Larvae of six different European non-target fly species and the target $D$. suzukii were exposed either on diet or blueberries to three Asian larval parasitoids, Asobara japonica, Leptopilina japonica, and Ganaspis cf. brasiliensis, and one European species, Leptopilina heterotoma. Asobara japonica showed the lowest specificity, attacking and developing in all Drosophilidae. Leptopilina japonica successfully parasitized two non-target Drosophilidae, D. melanogaster and D. subobscura, with one singly progeny emerging from D. immigrans. Ganaspis cf. brasiliensis had the highest level of specificity but variations occurred between two geographical populations tested. A Japanese population was strictly specific to $D$. suzukii, whereas another population from China parasitized $D$. suzukii, D. melanogaster and sporadically $D$. subobscura. The European L. heterotoma successfully developed in D. melanogaster, D. subobscura and occasionally in $D$. immigrans, but nearly all eggs and larvae in $D$. suzukii were encapsulated. These results show that Ganaspis cf. brasiliensis is the species with the highest potential for biological control, but more studies are needed on its taxonomic status and the existence of biotypes or cryptic species varying in their specificity before field releases can be conducted in Europe.
\end{abstract}

Keywords Spotted wing drosophila $\cdot$ Non-target effects $\cdot$ Host range $\cdot$ Fruit flies $\cdot$ Larval parasitoids

\section{Key message}

- Drosophila suzukii is a serious pest of soft fruit crops worldwide and classical biological control may provide an alternative to the use of chemical insecticides.

Communicated by M. Traugott.

Electronic supplementary material The online version of this article (https://doi.org/10.1007/s10340-018-1003-z) contains supplementary material, which is available to authorized users.

Tim Haye

t.haye@cabi.org

1 CABI, Delémont, Switzerland

2 Laboratory of Fundamental and Applied Research in Chemical Ecology (FARCE), Faculté des Sciences, University of Neuchâtel, Neuchâtel, Switzerland
- Host specificity tests were conducted with three Asian and one European parasitoid species, using five European Drosophila spp. and one Tephritidae species as hosts.

- A population of Ganaspis cf. brasiliensis from Tokyo (Japan) showed the highest degree of host specificity and is the best candidate for classical biological control.

\section{Introduction}

Globalization and climate change speed up the spread of new invasive pests, causing an estimated agricultural loss of more than \$1.4 trillion per year worldwide (Pimentel et al. 2001). Since the second half of the twentieth century, around $30 \%$ of alien arthropod species had established in Europe have originated from Asia (Roques et al. 2009). Among these, Drosophila suzukii Matsumura (Diptera, Drosophilidae), or spotted wing drosophila. This fly of East Asian origin was first found in 2008 in Europe and North America and, in just a few years, it invaded several continents (Asplen et al. 2015; Fraimout 
et al. 2017) and became a very serious pest of many fruit crops worldwide as non-crop or ornamental fruits (Walsh et al. 2011; Lee et al. 2015; Kenis et al. 2016).

Current control methods rely on chemical insecticides or expensive and labour-intensive cultural practices (Haye et al. 2016). An alternative approach to control $D$. suzukii could be to use classical biological control, i.e. introducing natural enemies from the native range of the pest. Recent studies indicated that natural enemies from Asia may provide a better area-wide control of $D$. suzukii's populations in the invaded areas than larval parasitoids from Europe and North America [e.g. Leptopilina heterotoma Thompson (Hymenoptera, Figitidae)] that are rarely able to complete their development in D. suzukii (Chabert et al. 2012; Poyet et al. 2013; Gabarra et al. 2015; Rossi-Stacconi et al. 2015; Daane et al. 2016). In Europe, only the generalist pupal parasitoids such as Trichopria drosophilae Perkins (Hymenoptera: Diapriidae) and Pachycrepoideus vindemmiae Rondani (Hymenoptera: Pteromalidae) can successfully develop in D. suzukii and T. drosophilae is considered for augmentative biocontrol, but its efficacy to control $D$. suzukii populations in infested orchards remains to be shown (Knoll et al. 2017; Rossi-Stacconi et al. 2017). Compared to chemical control, classical biological control, when applied properly, is often less harmful to the environment, shows direct benefits for food safety and provides effective long-term control of invasive species. However, potential adverse ecological effects have to be considered prior to the introduction of an exotic biological control agent (Heimpel and Mills 2017). Occasionally, introduced parasitoids and predators have unintended non-target impacts (van Driesche and Hoddle 2016) and, as a consequence, regulatory requirements have become more strict. In most countries, approval for release of classical biological control agents requires risk assessments, including detailed studies on host specificity (Hunt et al. 2008; Mason et al. 2013). Current practices usually include laboratory host specificity tests as a first step to define the fundamental host range of the potential biological control agent (van Driesche and Murray 2004; Bigler et al. 2006; van Lenteren et al. 2006).

Here we present new insights into the host specificity of three larval parasitoids that attack $D$. suzukii in its native range in Asia, Leptopilina japonica Novkovic \& Kimura, Ganaspis cf. brasiliensis (Ihering) (Hymenoptera, Figitidae) and Asobara japonica Belokobylskij (Hymenoptera, Braconidae), by testing them on a variety of potential European hosts.

\section{Materials and methods}

\section{Insect rearing}

\section{Target and non-target species}

The starting colony of $D$. suzukii was obtained from fruits of Rubus sp. and Fragaria sp. collected from various sites in Switzerland in 2015. Around five hundred adult flies were reared in gauze cages (BugDorm-4F4545@) kept at $22 \pm 2{ }^{\circ} \mathrm{C}, 60 \pm 10 \% \mathrm{RH}$ and a photoperiod of $16: 8 \mathrm{~h}(\mathrm{~L}: D)$. Flies were fed with sugar water provided on dental cotton rolls and wet cellulose paper was provided as additional water source. Tubes filled with $10 \mathrm{~g}$ of commercial artificial fly diet (Formula 4-24 medium $\odot$, Carolina Biological SupplyCo. Burlington, NC), $40 \mathrm{~mL}$ of $1.43 \mathrm{~g} \mathrm{~L}^{-1}$ of methyl-4-hydroxylbenzoate (inhibitor of fungal growth) and a pinch of yeast were placed in each cage as a food source and oviposition substrate. Tubes with $D$. suzukii eggs were changed twice a week and placed in incubators at $22{ }^{\circ} \mathrm{C}, 16 \mathrm{~h}$ light until emergence of adults, which were then randomly distributed among the rearing cage.

The selection of non-target hosts was based on phylogenetic relatedness, sympatry of target and non-target species and information on the geographical distribution and occurrence of European Drosophilidae available from the literature (FlyBase database, NCBI Taxonomy database; Kuhlmann et al. 2006; Fleury et al. 2009) (Fig. 1). In total, five European Drosophila spp. (D. busckii Coquillett, D. hydei Sturtevant, D. immigrans Sturtevant, D. melanogaster Meigen and D. subobscura Collin) and one Tephritidae species [Ceratitis capitata (Wiedemann)] were selected as non-target test species. The latter was selected as an out-group species as it is also able to oviposit into fresh berries as $D$. suzukii, in contrast to other European Drosophila spp. that usually attack decaying fruits and other organic matters. All non-target species were obtained from laboratory colonies of INRA, Sophia-Antipolis, France, in 2015. The Drosophila spp. were reared on the same artificial diet and under the same conditions as $D$. suzukii. Ceratitis capitata was reared on a homemade artificial diet (10 g of Carolina $\odot$ artificial fly diet, $10 \mathrm{~g}$ of carrot powder and $10 \mathrm{~g}$ of yeast powder with $40 \mathrm{~mL}$ of $1.43 \mathrm{~g} \mathrm{~L}^{-1}$ of methyl-4-hydroxylbenzoate) in cages of two hundred adult flies $(30 \times 30 \times 30 \mathrm{~cm}$ BugDorm-1@) .

\section{Parasitoid species}

The following parasitoids were collected from fruits infested with D. suzukii in Asia in 2015 (Girod et al. 2018b) and reared in the quarantine facilities of CABI 


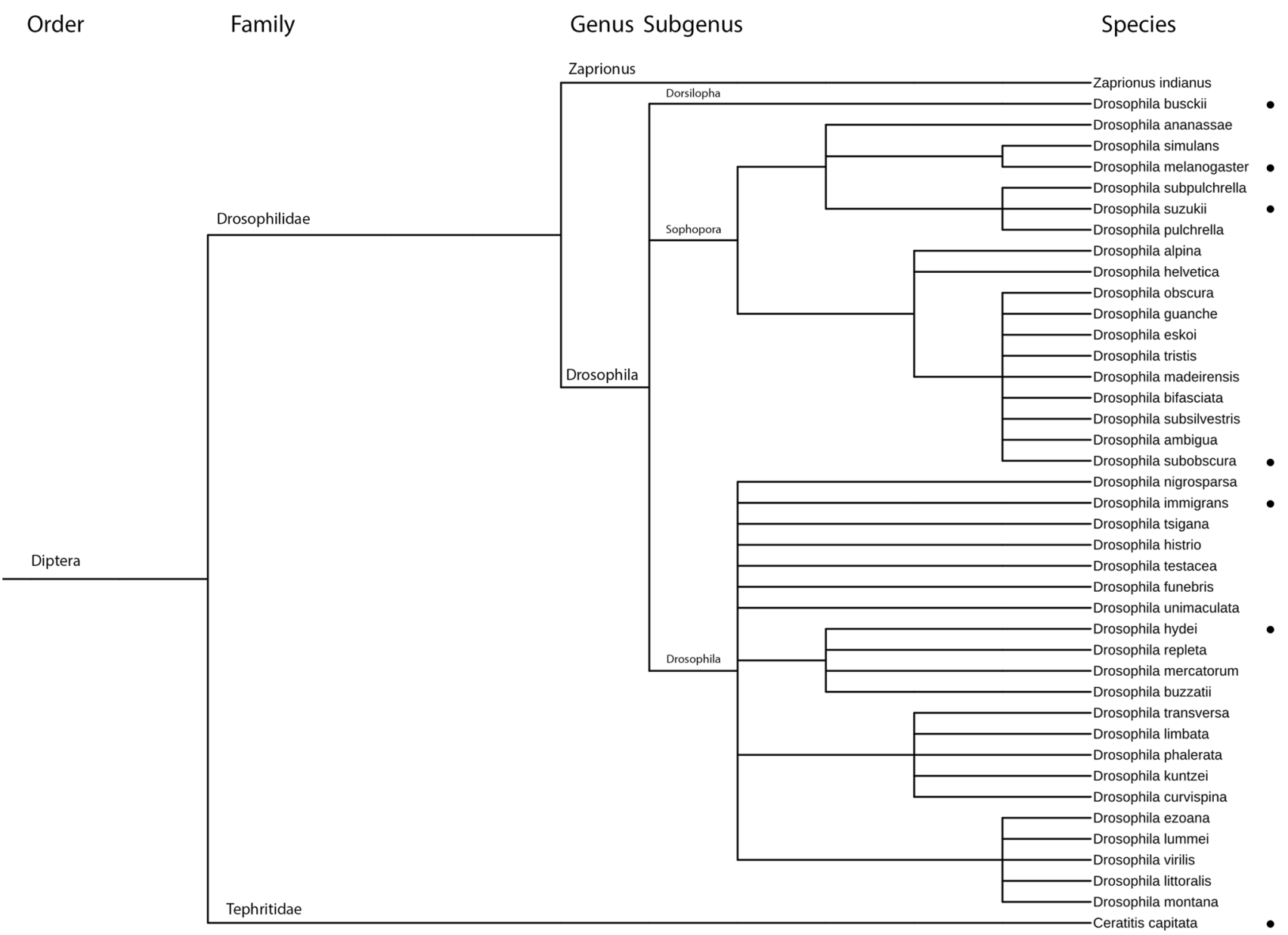

Fig. 1 Phylogenetic tree of European Drosophila spp. with tested species marked with dots. Ceratitis capitata and three exotic Drosophilidae attacking fresh fruits, Zaprionus indianus, Drosophila pulchrella

in Delémont, Switzerland: (1) Ganaspis cf. brasiliensis from Prunus cerasoides D. Don, (Kunming, Yunnan, China) and (2) Ganaspis cf. brasiliensis Prunus serrulata Lindl. (Tokyo, Japan); (3) Leptopilina japonica from $P$. cerasoides (Kunming, Yunnan, China); (4) Leptopilina japonica from Prunus sp. (Beijing, China); (5) Asobara japonica Belokobylskij collected by sweeping over $P$. serrulata fruits (Tokyo, Japan). Ganaspis brasiliensis, which has been recorded from various continents (Buffington and Forshage 2016; Nomano et al. 2017), is likely a complex of cryptic species with different distributions and various degrees of specificity (Nomano et al. 2017). Until the taxonomic status of $G$. brasiliensis has been clarified with molecular methods, the two populations from China and Japan used in this study are referred to as Ganaspis cf. brasiliensis. In addition to the Asian species, we also tested the European L. heterotoma, which was collected in Delémont, Switzerland, in summer 2015. and Drosophila subpulchrella, were added to the tree. The tree is based on Fauna Europaea and the phylogenetic tree was built with NCBI Taxonomy database, Software iTOL (Letunic and Bork 2016)

The Asian Figitidae were reared in boxes ( $\varnothing$ $90 \times 50 \mathrm{~mm}$ ), each containing ca. 50-60 individuals and kept at $22 \pm 2{ }^{\circ} \mathrm{C}, 60 \pm 10 \% \mathrm{RH}$ and $16 \mathrm{~h}$ light. An Eppendorf tube with a wet cellulose paper was added to each rearing box as a water source. Boxes were closed with a foam plug on which a drop of honey was placed as a food source. Fresh blueberries (Vaccinium corymbosum) were placed in each D. suzukii rearing cage for $48 \mathrm{~h}$ for infestation, and then distributed among the parasitoid rearing boxes for another $48 \mathrm{~h}$ to allow female parasitoids to oviposit in the young fly larvae. After the exposure infested fruits were removed and kept in rearing tubes $(\varnothing$ $50 \times 100 \mathrm{~mm}$ ) with a filter paper at the bottom to absorb leaking fruit juice. The thelytokous species $A$. japonica and the European L. heterotoma were maintained offering the wasps first instar larvae of $D$. melanogaster in artificial diet for 3-4 days. A drop of honey was added to each tube as food source. The rearing tubes were checked daily 
for newly emerged parasitoids, which were immediately transferred to the rearing boxes.

\section{Host specificity testing}

Before the host specificity tests, the oviposition substrates containing the fly larvae had to be modified due to differences in attractiveness to the parasitoid and fly species. In an earlier study it had been shown that the blended CAROLINA@ diet used for rearing the Drosophila spp. is not accepted as substrate by Ganaspis cf. brasiliensis (Girod et al. 2018a). However, besides D. suzukii, none of the Drosophila species used in this study lays eggs in fresh fruits. Consequently, a blended diet with fruit was developed, which was accepted by all the Drosophilidae, Tephritidae and parasitoids tested ( $25 \mathrm{~g}$ of blended fresh blueberry, $40 \mathrm{~mL}$ of $1.43 \mathrm{~g} \mathrm{~L}^{-1}$ of methyl-4-hydroxylbenzoate and $20 \mathrm{~g}$ of blended CAROLINA@ diet).

Specificity tests were carried out in two steps. In experiment A, A. japonica, L. japonica and L. heterotoma were tested on D. suzukii, D. melanogaster, D. immigrans, D. subobscura and D. busckii in plain regular CAROLINA@ diet instead of fruit (Table 1, Experiment A), because Girod et al. (2018a) demonstrated that these species would show the same parasitism behaviour with larvae in this artificial diet and blueberries. In experiment B, the two populations of Ganaspis cf. brasiliensis were tested (1) on the same host species as in experiment $\mathrm{A}$ in the modified diet containing blended blueberries, and (2) on D. suzukii and C. capitata in fresh blueberries (Table 1, Exp. B). Finally, in experiment B, $L$. japonica was also tested on D. hyde $i$ and $C$. capitata (two species that were not assessed in experiment A). Asobara japonica was not assessed further because experiment A had shown that this species is highly polyphagous. In total, 43 different combinations of parasitoids, oviposition substrates and host were tested (Table 1). All experiments were conducted under laboratory conditions at $22 \pm 2{ }^{\circ} \mathrm{C}, 60 \pm 10 \%$ $\mathrm{RH}$ and a photoperiod of 16:8 $\mathrm{h}(L: D)$.

From the laboratory colonies, 0-12-h-old female parasitoids were collected and placed in tubes with males (sex ratio female/male 2:1) for $72 \mathrm{~h}$ to ensure that they were mated and mature at the time of the experiments (Girod et al. 2018a). After 3 days, single female parasitoids were exposed for a period of $48 \mathrm{~h}$ to 10-30 Drosophila spp. larvae that were 12-20 h old. Drosophila spp. show small differences in their development time and exposing the hosts for $48 \mathrm{~h}$ ensured that all Drosophila spp. were in their first instar and early second instars, the suitable stages for larval parasitoids of Drosophila spp. (Carton et al. 1986). Larvae of C. capitata were used for experiment after a period of incubation of 24-32 h. At this time larvae had reached the 1 st instar and had a similar size as Drosophilidae larvae.

After 2 days of exposure, single female parasitoids were removed. A total of 30 replicates (60 for D. suzukii and D. busckii in experiment B) and 20 controls (fly larvae without exposure to parasitoids) were performed for each species. Fly and parasitoid emergence was checked daily and all emerging individuals were counted and sexed. The number of flies with encapsulated parasitoid eggs or larvae was recorded under a microscope after squeezing the fly between two microscope glass slides. The few female parasitoids that died during the experiments were excluded from the analysis, as were the tubes without any emergence of flies or parasitoids. Since host attacks by larval parasitoids may result in failed parasitoid offspring development, while causing the host larva to die (see and

Table 1 Experimental testing scheme for each parasitoid, diet, and host (*only tested hosts of L. japonica Beijing, China for experiment B)

\begin{tabular}{|c|c|c|c|c|c|}
\hline \multirow[t]{2}{*}{ Experiment \# } & \multicolumn{2}{|l|}{ Parasitoids } & \multirow[t]{2}{*}{ Oviposition substrate } & \multirow[t]{2}{*}{ Hosts } & \multirow{2}{*}{$\begin{array}{l}\text { \# Repli- } \\
\text { cates }(n)\end{array}$} \\
\hline & Species & Origin & & & \\
\hline \multirow{5}{*}{ A } & \multirow{5}{*}{$\begin{array}{l}\text { Asobara japonica } \\
\text { Leptopilina japonica } \\
\text { Leptopilina japonica } \\
\text { Leptopilina heterotoma }\end{array}$} & \multirow{5}{*}{$\begin{array}{l}\text { Tokyo, Japan } \\
\text { Beijing, China } \\
\text { Kunming, China } \\
\text { Delémont, Switzerland }\end{array}$} & \multirow[t]{5}{*}{ Artificial diet } & D. melanogaster & 30 \\
\hline & & & & D. busckii & 30 \\
\hline & & & & D. subobscura & 30 \\
\hline & & & & D. immigrans & 30 \\
\hline & & & & D. suzukii & 30 \\
\hline \multirow[t]{9}{*}{ B } & \multirow{9}{*}{$\begin{array}{l}\text { Ganaspis cf. brasiliensis } \\
\text { Ganaspis cf. brasiliensis } \\
\text { Leptopilina japonica* }\end{array}$} & \multirow{9}{*}{$\begin{array}{l}\text { Kunming, China } \\
\text { Tokyo, Japan } \\
\text { Beijing, China }\end{array}$} & \multirow{7}{*}{$\begin{array}{l}\text { Artificial diet }+ \text { blended } \\
\text { blueberry }\end{array}$} & D. hydei* & 30 \\
\hline & & & & D. melanogaster & 30 \\
\hline & & & & D. busckii & 60 \\
\hline & & & & D. subobscura & 30 \\
\hline & & & & D. immigrans & 30 \\
\hline & & & & D. suzukii ${ }^{*}$ & 60 \\
\hline & & & & C. capitata* & 30 \\
\hline & & & \multirow[t]{2}{*}{ Blueberry } & D. suzukii* & 60 \\
\hline & & & & C. capitata* & 30 \\
\hline
\end{tabular}


references Abram et al. 2016 therein), emergence from controls (flies) was compared to emergence from treatments (flies + parasitoids). In an earlier study it was already shown that parasitoids did not lead to a significant increase of larval mortality of $D$. suzukii when hosts were exposed in blueberries (Girod et al. 2018a).

For each specificity test with different parasitoid populations or substrates, a series of parameters was measured: the number of emerged flies $\left(n_{\mathrm{d}}\right)$, the number of emerged flies with an encapsulated parasitoid egg $\left(n_{\mathrm{e}}\right)$ among the total number of emerged flies $\left(n_{\mathrm{d}}\right)$, the number of emerged parasitoids $\left(n_{\mathrm{p}}\right)$, the total number of emerged individuals $\left(n=n_{\mathrm{d}}+n_{\mathrm{p}}\right)$, and the number of female parasitoids that attacked fly larvae [i.e. wasps for which at least one emerging parasitoid or encapsulated egg was counted $\left.\left(n_{\mathrm{o}}\right)\right]$.

Five parameters of the host-parasitoid interaction were measured for each population and condition:

1. The "Proportion of Ovipositing Females" (POF) corresponds to the number of female parasitoids which had laid at least one egg in fly larvae $\left(n_{\mathrm{o}}\right)$ divided by the number of females tested $(N)$. It was calculated as $\mathrm{POF}=n_{\mathrm{o}} / N$.

2. The "Overall Parasitism Rate" (OPR), which is the proportion of parasitized hosts, i.e. the proportion of flies that contained an encapsulated egg or produced parasitoid offspring. It was calculated as OPR $=\left(n_{\mathrm{p}}+n_{\mathrm{e}}\right) / n$ for each parasitoid female.

3. The "Apparent Parasitism Rate" (APR), which is the proportion of parasitoid offspring among the total number of insects that emerged. It was calculated as $\mathrm{APR}=n_{\mathrm{p}} / n$ for each parasitoid female.

4. The "Encapsulation Rate" (ER) corresponds to the proportion of emerged flies that contained encapsulated parasitoid eggs or larvae in their abdomens which they had carried over from the parasitized larval stage. It was calculated as ER $=n_{\mathrm{e}} /\left(n_{\mathrm{p}}+n_{\mathrm{e}}\right)$ for each parasitoid female.

5. The "Encapsulation Level" (EL), which is estimated as the proportion of emerged flies that contained a capsule among the total number of insects that emerged. EL was calculated as $\mathrm{EL}=n_{\mathrm{e}} / n$ for each parasitoid female.

\section{Statistical analysis}

Values of OPR and APR for each species and condition were compared with generalized linear models (Hoffmeister et al. 2006). Since our results were a mixture of zeros and positive values, we used a Tweedie family (Poisson distribution), followed by pairwise comparisons, using the Tukey's post hoc test. All statistical analyses were performed with the $\mathrm{R}$ studio software (version 3.3.3) (R Core Team 2017).

\section{Results}

Females of the European parasitoid, L. heterotoma, attacked all tested hosts in artificial diet, except $D$. busckii. The proportion of ovipositing females (POF) ranged from 16.7 to $80.0 \%$ (Fig. 2a). While this parasitoid managed to produce offspring on D. melanogaster and D. subobscura with successful parasitism (APR) of 46.7 and $30.4 \%$, respectively, its APR was extremely low $(<1 \%)$ on D. immigrans and $D$. suzukii (Fig. 3a). Only three females produced offspring on D. immigrans. From D. suzukii larvae, only a single parasitoid emerged and the encapsulation rate (ER) was as high as 99.4\%. In total, around 30\% of the emerged flies contained a capsule (EL) in their abdomen (ESM 1). Surprisingly, $L$. heterotoma, was highly attracted to D. suzukii with $73.3 \%$ of the females laying eggs (POF) in the D. suzukii larvae.

The two L. japonica populations (Beijing and Kunming) showed similar responses to non-target hosts. The proportions of females from the Beijing population that laid eggs (POF) on D. melanogaster and D. suzukii were high, i.e. 51.9 and $63.3 \%$ respectively, whereas it was 40.0 and $31.0 \%$ for the Kunming population. Lower proportions were observed on D. subobscura $(7.4$ and $3.7 \%$ for the Beijing population and the Kunming population) and $D$. immigrans (6.7 and 7.1\%) (Fig. 2a). Successful parasitism (APR) for the Beijing population on D. melanogaster and D. suzukii was high, i.e. 36.3 and $35.17 \%$ respectively, and 21.9 and $15.0 \%$ for the Kunming population. APR was much lower on D. subobscura ( $4.3 \%$ for the Beijing population and $2.8 \%$ for the Beijing population) and only one progeny emerged from $D$. immigrans (APR $=0.1 \%$ in the Beijing population) (Fig. 3a). Leptopilina japonica females did not attack $D$. busckii in artificial diet. Ceratitis capitata larvae were parasitized neither in blended diet nor in fresh blueberries. However, on D. suzukii, the proportions of females laying eggs on blended diet and blueberries were 63.0 and $61.9 \%$, respectively. In both conditions, successful parasitism was high, reaching 33.1 and $24.1 \%$ respectively (Figs. 2b, 3b). Encapsulation levels were low for the two L. japonica populations in all parasitized hosts.

Asobara japonica females successfully attacked all tested European fly species and D. suzukii with a proportion of ovipositing females (POF) ranging from 12.0 to $85.7 \%$ (Fig. 2a). Of all tested parasitoids, it showed the highest successful parasitism (APR) on D. melanogaster with $54.3 \%$, D. busckii with $2.9 \%$, D. subobscura with $38.7 \%$, D. immigrans with $1.2 \%$ and D. suzukii with $58.1 \%$ in artificial diet (Fig. 3a). Asobara japonica eggs and larvae were rarely encapsulated except on $D$. immigrans $(\mathrm{ER}=33.3 \%)$. Of all parasitoids tested, A. japonica was most attracted to $D$. suzukii, with $85.7 \%$ of the females laying eggs (POF) in the D. suzukii larvae. 


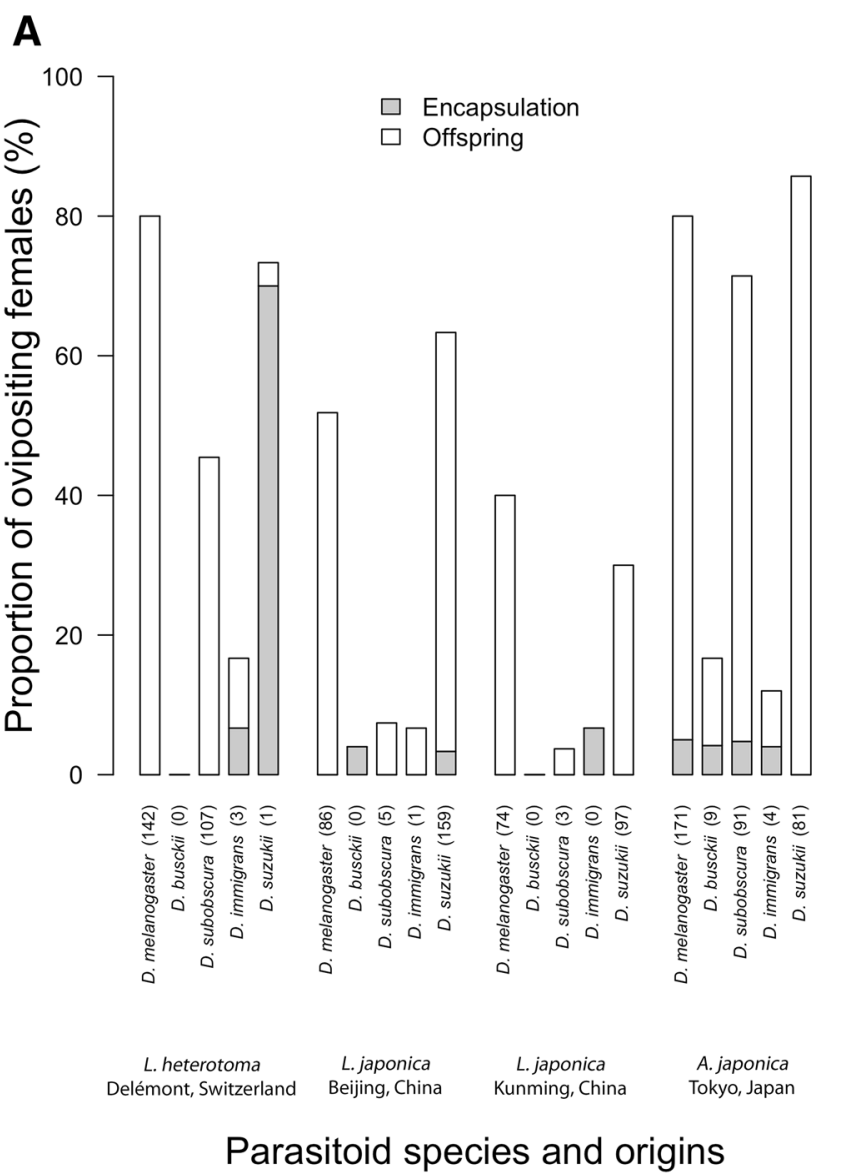

Fig. 2 Proportion of ovipositing parasitoid females when exposed to larvae of various hosts in a CAROLINA $\odot$ diet and $\mathbf{b}$ blueberries or mixed, blended diet. Oviposition was recorded as successful when

Both Ganaspis cf. brasiliensis populations hardly attacked larvae of D. hydei, D. immigrans and C. capitata, and no progeny emerged from these hosts (Figs. 2b, 3b). Overall, the proportion of females that oviposited (POF) was higher for the Kunming population (ranging from 3.3 to $74 \%$ ) than the Tokyo population (ranging from 3.5 to $46.2 \%$ ) (Fig. 2b). No emergence of parasitoids was observed for the Ganaspis cf. brasiliensis population from Tokyo on D. melanogaster and D. subobscura, and the few attempts on the later host $(\mathrm{OPR}<1 \%)$ were all encapsulated $(\mathrm{ER}=100 \%)$. In contrast, Ganaspis cf. brasiliensis from Kunming did manage to develop in high numbers on D. melanogaster, and two specimens emerged from $D$. subobscura, with ER $14.8 \%$ and $25.0 \%$, respectively. Even on its natural host, i.e. D. suzukii, Ganaspis cf. brasiliensis (Tokyo population) rarely successfully developed on blended diet, with APR as low as $0.2 \%$ and ER of $85.0 \%$; however, in blueberry, APR was up to $19.6 \%$ and ER was down to $15.6 \%$. The Kunming population performed much better on $D$. suzukii in blended diet, with APR of $15.98 \%$ and ER of $18.3 \%$. In blueberry, APR was $32.5 \%$ and ER was $15.1 \%$ (Fig. 3 b).

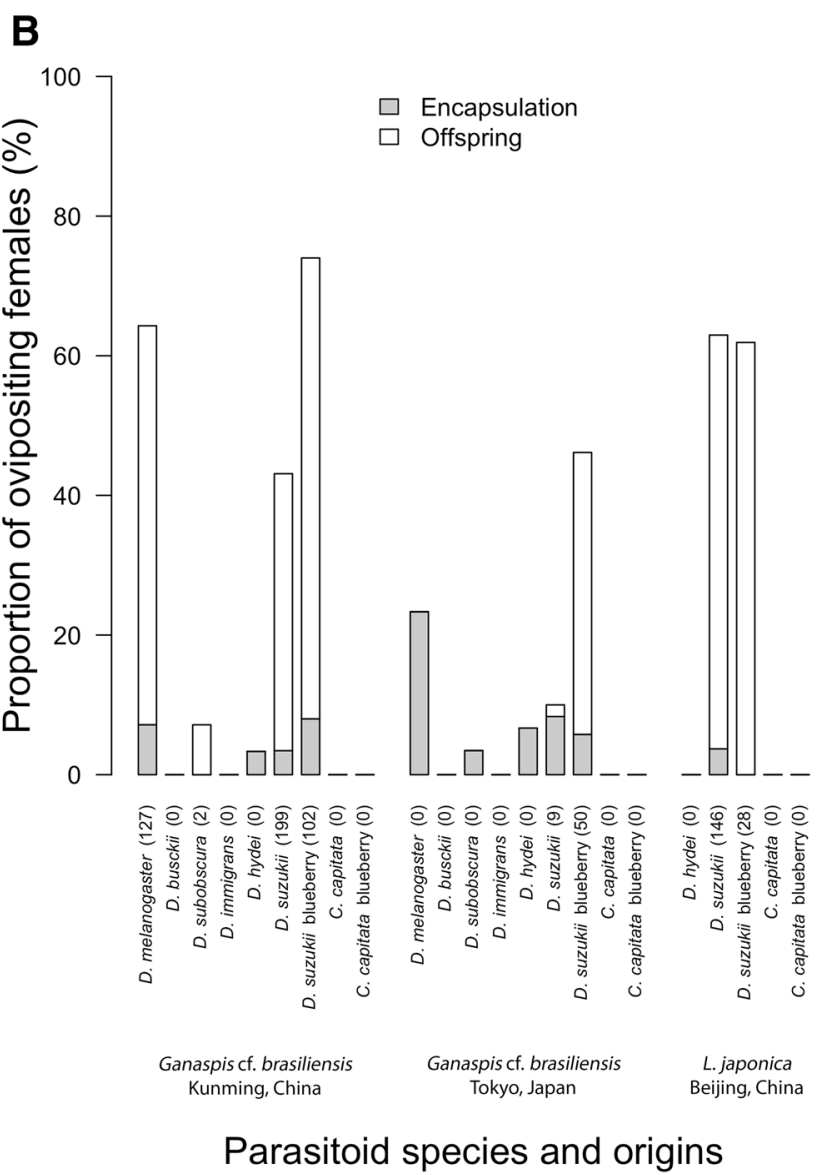

either an encapsulated egg or larva was found in the abdomen of the emerged fly or when offspring was produced (in brackets, total number of parasitoid offspring)

For none of the parasitoids there were indications that host attacks resulted in increased larval mortality without parasitoid development (ESM2). However, in some cases counting the fly eggs laid in artificial diet accurately was not possible, resulting in higher numbers of emerged individuals than eggs counted at the beginning of the experiment. For these cases, we could not perform a comparison, but the high numbers of emerged individuals suggested no or very low larval mortality.

\section{Discussion}

Among the Asian parasitoids tested, the Ganaspis cf. brasiliensis population from Tokyo showed the highest degree of host specificity. Successful development was observed exclusively in $D$. suzukii in blueberries. Kasuya et al. (2013a) obtained exactly the same results with a population from the same locality [as 'suzukii-specialized' type of Ganaspis xanthopoda (Kasuya et al. 2013a)]. They carried out laboratory tests and showed that Ganaspis cf. brasiliensis parasitized 


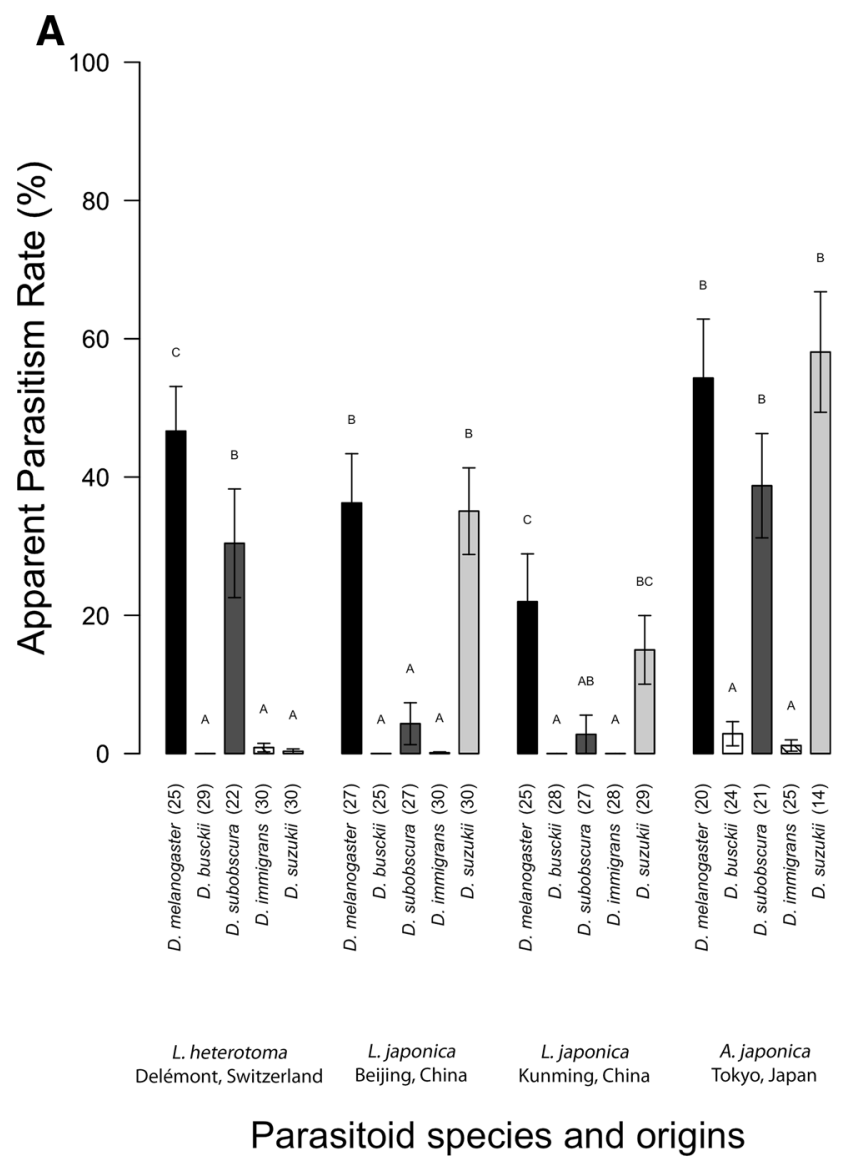

Fig. 3 Mean successful parasitism (APR) ( \pm SE) caused by parasitoids exposed to larvae of various hosts in a CAROLINA@ diet and b blueberries or mixed, blended diet. APR was calculated as the proportion of parasitoid emergence among the total number of insects that emerged (in brackets: number of replicates $=$ females included in the calculation). For each parasitoid and experiment, bars with

D. suzukii larvae in fresh cherry fruits, but did not parasitize those in a Drosophila artificial diet. In addition, they did not parasitize larvae of the following species: Drosophila lutescens Okada, D. rufa Kikkawa \& Peng, D. auraria Peng, D. biauraria Block \& Wheeler and D. triauraria Block \& Wheeler even when these occurred in fresh cherry fruits. However, too few replicates were made on these species to draw firm conclusions regarding their suitability as hosts. Surprisingly, in our study, the Kunming population of the supposedly similar parasitoid species showed less specificity, as it very successfully parasitized $D$. melanogaster and $D$. suzukii in the blended diet, whereas in earlier trials, we had failed to rear the same population in an artificial diet without fruits (Girod et al. 2018a). Recent work by Nomano et al. (2017) has shown that the G. brasiliensis complex, to which the tested Ganaspis cf. brasiliensis belongs, includes several cryptic species with totally different host ranges (see also Kasuya et al. 2013b). It is possible that additional

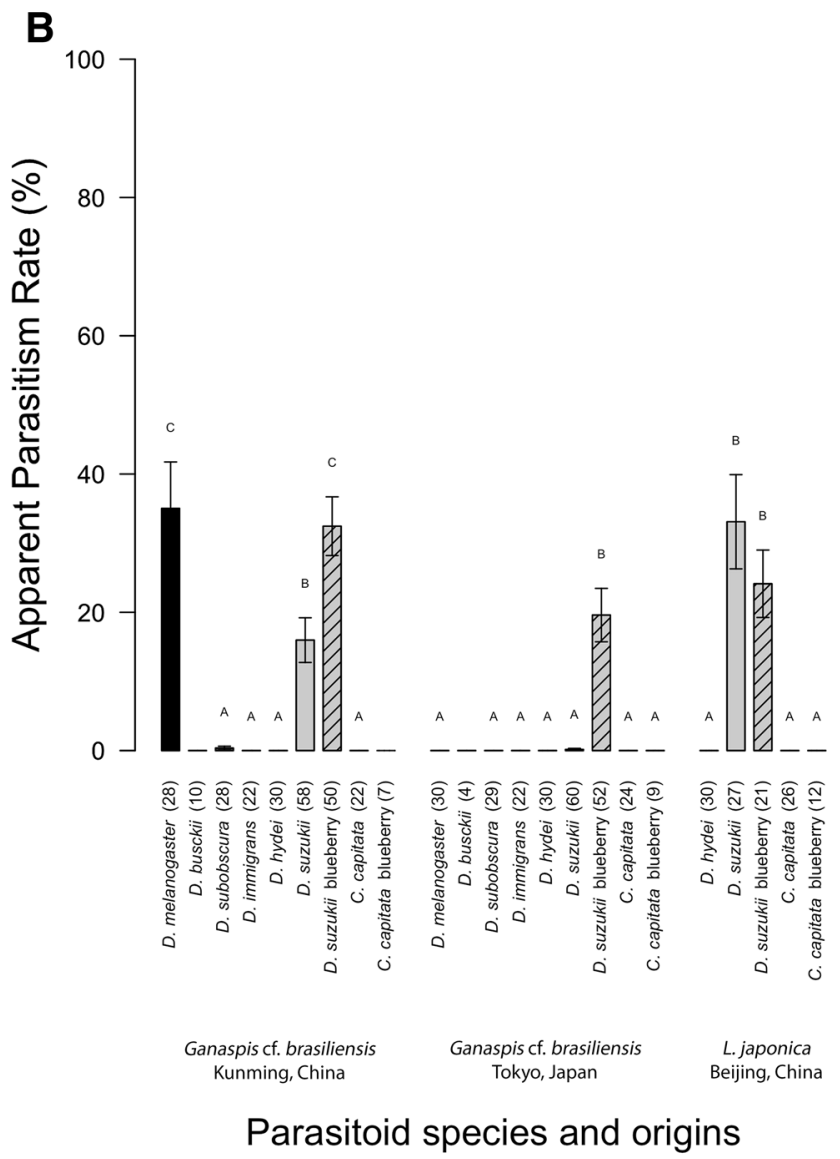

the same letters indicate no significant differences between treatments (GLM (Tweedie family) Tukey post hoc, $p \leq 0.05$ ). Tests on $D$. busckii in experiment B were not included in the analyses because of the low number of replicates due to the absence of fly offspring in most tubes

cryptic species or biotypes varying in their specificity occur even within the $G$. brasiliensis group that parasitizes $D$. suzukii. Intraspecific variations in host preference or even host specificity are rather common in parasitoids (Höller 1991; Vazquez et al. 2004). It is therefore of upmost importance to improve our understanding of the taxonomy of this group in relation to its specificity before using Ganaspis $\mathrm{cf}$. brasiliensis in a biological control programme, in order to choose the most suitable and specific population. The same Ganaspis cf. brasiliensis is the main larval parasitoid of $D$. suzukii in South Korea (Daane et al. 2016), Japan (Kasuya et al. 2013a; Wachi et al. 2015; Matsuura et al. 2017) and China (Girod et al. 2018b), where it probably also attacks two other fruit-inhabiting drosophilids, D. pulchrella and $D$. subpulchrella, the two sister species of $D$. suzukii.

In contrast to what was observed for the two Ganaspis cf. brasiliensis populations, no difference was found between the two populations of $L$. japonica in terms of their degree of 
specificity. Both populations happily attacked and developed in larvae of D. melanogaster, D. subobscura and D. suzukii in all substrates but not in the four other hosts, with the exception of one successful development in a $D$. immigrans larva. Leptopilina japonica frequently parasitized larvae of D. suzukii in Japan (Novković et al. 2011; Matsuura et al. 2017), South Korea (Daane et al. 2016) and China, where it probably attacks also larvae of D. pulchrella and D. subpulchrella (Girod et al. 2018b). In Japan, it is also found on $D$. biauraria and $D$. rufa and it has been successfully reared on D. simulans in the laboratory (Novković et al. 2011). Thus, $L$. japonica is probably not sufficiently specific to be considered for introduction into Europe.

The third Asian parasitoid species tested in this study appeared to be the most polyphagous. Asobara japonica attacked all Drosophilidae proposed in the first experiment and was thus excluded from the following tests. This species was already known as a polyphagous parasitoid in Asia, being recorded on more than 25 Drosophilidae species (Nomano et al. 2015). Daane et al. (2016) and Guerrieri et al. (2016) recorded it on D. suzukii in South Korea and Mitsui and Kimura (2010), Nomano et al. (2015) and Matsuura et al. (2017) in Japan. Other studies in Europe and North America showed its ability to parasitize $D$. suzukii by affecting the haemocyte load of larvae, thereby overcoming its cellular immune system (Chabert et al. 2012; Kacsoh and Schlenke 2012; Poyet et al. 2013). Despite its abundance, A. japonica is rarely obtained from $D$. suzukii in Japanese fresh fruits, possibly because of its attraction for hosts in fermenting fruits, decayed mushrooms and plant leaves (Nomano et al. 2015). However, Biondi et al. (2017) showed that, in the laboratory, $A$. japonica females were able to learn exploiting volatiles emitted by fruits infested by $D$. suzukii. Nevertheless, its polyphagy excludes it from the list of potential candidates for introduction.

The European L. heterotoma showed a strong interest in D. suzukii larvae; however, the vast majority of parasitoid eggs and larvae were encapsulated by the host, confirming earlier studies (Chabert et al. 2012; Kacsoh and Schlenke 2012; Poyet et al. 2013; Knoll et al. 2017). Only one L. heterotoma progeny was able to overcome the immune response of D. suzukii and, although in some cases the rate of encapsulation avoidance may be much higher (e.g. 10-30\% in Rossi-Stacconi et al. 2015, 2017), parasitism by L. heterotoma has not yet been found in the field (Kenis et al. 2016). However, it cannot be ruled out that over time L. heterotoma or other native larval parasitoid can become adapted to the new host, as observed in many other cases (Henter and Via 1995; Urbaneja et al. 2000; Jones et al. 2015). Although it was reported in early studies that $D$. subobscura larvae were found to be unable to form a capsule around eggs of the parasitoids A. tabida and L. heterotoma (Eslin and Doury 2006), capsules were sporadically found in the abdomens of
D. subobscura that had been parasitized at the larval stage by Ganaspis cf. brasiliensis (Kunming strain: 1 capsule, Tokyo strain: 2 capsules, ESM 1). Since Eslin and Doury (2006) did not test any Ganaspis spp., it remains unclear though whether these rare encapsulations were related to the parasitoid species or the D. subobscura strain used in our experiments.

So far, Ganaspis cf. brasiliensis appears to be the best candidate for introduction into Europe and other invaded regions. It is the main parasitoid of D. suzukii in East Asia (Daane et al. 2016; Matsuura et al. 2017; Girod et al. 2018b), and this study showed that it has the narrowest host range. However, our observations that specificity varies with populations implies that further studies are needed to elucidate mechanisms leading to specificity and to investigate the existence of cryptic species or geographic biotypes showing difference in host location, host preference, searching and oviposition behaviour. In addition, more Drosophilidae could be tested with Ganaspis cf. brasiliensis, however, this study and Girod et al. (2018a) highlighted the difficulty of finding a substrate that is suitable for both Ganaspis cf. brasiliensis and the non-target species. Ganaspis cf. brasiliensis prefers ovipositing in fruits, and even a diet with blended fruits appeared not suitable for at least one of the two populations tested. In contrast, most Drosophilidae live in decaying plants and fungal material (van Alphen and Janssen 1981), and will not attack fresh or even rotting fruits and, thus, cannot be tested with Ganaspis cf. brasiliensis. Besides, showing that a certain biotype of Ganaspis cf. brasiliensis is specific to larvae in fresh fruits is a strong argument in favour of its probable specificity in regions of introductions where no native Drosophilidae live in fresh fruits. However, prior to releases the taxonomic status of Ganaspis cf. brasiliensis needs to be clarified and additional tests are needed to proof its efficiency on other fruits and in nature.

\section{Author contributions}

PG, MK and TH conceived research. PG, OL and TU conducted experiments. PG, MK and TH wrote the manuscript. All authors edited the manuscript and approved the final version.

Acknowledgements The research leading to these results received funding from the European Union's Seventh Framework programme for research, technological development and demonstration under Grant Agreement Number 613678 (DROPSA), the Swiss Federal Office for the Environment and the Loterie Romande. We would like to thank Nicolas Ris (INRA Sophia-Antipolis, France), Jinping Zhang and Renya Liao (MoA-CABI Joint Laboratory for Biosafety, Beijing, China), Hao Wu, Guohua Chen, Yan Liu, Yan Xiong, Chun Xiao (Yunnan Agricultural University, Kunming, China) and Masahito T. Kimura (University of Hokkaido, Japan) for helping with the parasitoid collections. We thank S. Fischer (Agroscope Changins, Switzerland) for 
providing the D. suzukii colony. We are grateful to Romain Angeleri, Gaëlle Beureux, Benoit Poltera and Laureline Rossignaud for their assistance with experiments and maintaining insect colonies. We thank Matthew L. Buffington (Systematic Entomology Laboratory, USDAARS c/o NMNH, Smithsonian Institution, Washington, USA) for the identification of the Figitidae and Alexandre Aebi, Catherine Baroffio, Antonio Biondi and Patrik Kehrli for their advice and comments on earlier versions of this manuscript. CABI is an international intergovernmental organization, and we gratefully acknowledge the core financial support from our member countries (and lead agencies) including the United Kingdom (Department for International Development), China (Chinese Ministry of Agriculture), Australia (Australian Centre for International Agricultural Research), Canada (Agriculture and Agri-Food Canada), Netherlands (Directorate-General for International Cooperation), and Switzerland (Swiss Agency for Development and Cooperation). See http://www.cabi.org/about-cabi/who-we-work-with/ key-donors/for full details.

\section{Compliance with ethical standards}

Conflict of interest The authors have declared that no conflict of interest exists.

Informed consent Informed consent was obtained from all individual participants included in the study.

Research involving human participants and/or animals This article does not contain any studies with human participants or animals (vertebrates) performed by any of the authors.

Open Access This article is distributed under the terms of the Creative Commons Attribution 4.0 International License (http://creativeco mmons.org/licenses/by/4.0/), which permits unrestricted use, distribution, and reproduction in any medium, provided you give appropriate credit to the original author(s) and the source, provide a link to the Creative Commons license, and indicate if changes were made.

\section{References}

Abram PK, Brodeur J, Burte V, Boivin G (2016) Parasitoid-induced host egg abortion: an underappreciated component of biological control services provided by egg parasitoids. Biol Control 98:52-60

Asplen MK, Anfora G, Biondi A et al (2015) Invasion biology of spotted wing Drosophila (Drosophila suzukii): a global perspective and future priorities. J Pest Sci 88:469-494. https://doi. org/10.1007/s10340-015-0681-z

Bigler F, Babendreier D, Kuhlmann U (2006) Environmental impact of invertebrates for biological control of arthropods: methods and risk assessment. CABI, Wallingford

Biondi A, Wang X, Miller JC et al (2017) Innate olfactory responses of Asobara japonica toward fruits infested by the invasive Spotted Wing Drosophila. J Insect Behav 30:495-506. https://doi. org/10.1007/s10905-017-9636-y

Buffington ML, Forshage M (2016) Redescription of Ganaspis brasiliensis (Ihering, 1905), new combination, (Hymenoptera: Figitidae) a natural enemy of the invasive Drosophila suzukii (Matsumura, 1931) (Diptera: Drosophilidae). Proc Entomol Soc Wash 118:1-13

Carton Y, Bouletreau M, van Lenteren JC, van Alphen JJM (1986) The Drosophila parasitic wasps. In: Ashburner M, Novitsky E (eds)
The genetics and biology of Drosophila, vol 3. Academic press, London, pp 347-389

Chabert S, Allemand R, Poyet M et al (2012) Ability of European parasitoids (Hymenoptera) to control a new invasive Asiatic pest, Drosophila suzukii. Biol Control 63:40-47. https://doi. org/10.1016/j.biocontrol.2012.05.005

Daane KM, Wang X-G, Biondi A et al (2016) First exploration of parasitoids of Drosophila suzukii in South Korea as potential classical biological agents. J Pest Sci 89:823-835. https://doi.org/10.1007/ s10340-016-0740-0

Eslin P, Doury G (2006) The fly Drosophila subobscura: a natural case of innate immunity deficiency. Dev Comp Immunol 30:977-983

Fleury F, Gibert P, Ris N, Allemand R (2009) Ecology and life history evolution of frugivorous Drosophila parasitoids. Adv Parasitol 70:3-44

FlyBase. http://flybase.org

Fraimout A, Debat V, Fellous S et al (2017) Deciphering the routes of invasion of Drosophila suzukii by means of $\mathrm{ABC}$ random forest. Mol Biol Evol 34:980-996

Gabarra R, Riudavets J, Rodríguez GA et al (2015) Prospects for the biological control of Drosophila suzukii. Biocontrol 60:331-339. https://doi.org/10.1007/s10526-014-9646-z

Girod P, Rossignaud L, Haye T et al (2018a) Development of Asian parasitoids in larvae of $D$. suzukii feeding on blueberry and artificial diet. J Appl Entomol. https://doi.org/10.1111/jen.12496

Girod P, Ris N, Borowiec N, Zhang J, Wu H, Fang Y, Chen G, Xiao C, Kimura MT, Buffington M, Peris-Felipo F, Aebi A, Haye T, Kenis M (2018b) Parasitoid complex of D. suzukii and other fruit feeding Drosophila spp. in Asia. Sci Rep (in press)

Guerrieri E, Giorgini M, Cascone P et al (2016) Species diversity in the parasitoid genus Asobara (Hymenoptera: Braconidae) from the native area of the fruit fly pest Drosophila suzukii (Diptera: Drosophilidae). PLoS ONE 11:e0147382

Haye T, Girod P, Cuthbertson AGS et al (2016) Current SWD IPM tactics and their practical implementation in fruit crops across different regions around the world. J Pest Sci 89:643-651

Heimpel GE, Mills NJ (2017) Biological control: ecology and applications. Cambridge University Press, Cambridge

Henter HJ, Via S (1995) The potential for coevolution in a host-parasitoid system. I. Genetic variation within an aphid population in susceptibility to a parasitic wasp. Evolution (N Y) 49:427-438. https://doi.org/10.1111/j.1558-5646.1995.tb02275.x

Hoffmeister TS, Babendreier D, Wajnberg E (2006) Statistical tools to improve the quality of experiments and data analysis for assessing non-target effects. In: Bigler F, Babendreier D, Kuhlman U (eds) Environmental impact of invertebrates for biological control of arthropods. CABI Publishing, Wallingford, pp 222-240

Höller CA (1991) Evidence for the existence of a species closely related to the cereal aphid parasitoid Aphidius rhopalosiphi De Stefani-Perez based on host ranges, morphological characters, isoelectric focusing banding patterns, cross-breeding experiments and sex pheromo. Syst Entomol 16:15-28

Hunt EJ, Kuhlmann U, Sheppard A et al (2008) Review of invertebrate biological control agent regulation in Australia, New Zealand, Canada and the USA: recommendations for a harmonized European system. J Appl Entomol 132:89-123

Jones TS, Bilton AR, Mak L, Sait SM (2015) Host switching in a generalist parasitoid: contrasting transient and transgenerational costs associated with novel and original host species. Ecol Evol 5:459-465. https://doi.org/10.1002/ece3.1333

Kacsoh BZ, Schlenke TA (2012) High hemocyte load is associated with increased resistance against parasitoids in Drosophila suzukii, a relative of D. melanogaster. PLoS ONE 7:e34721. https://doi. org/10.1371/journal.pone.0034721

Kasuya N, Mitsui H, Aotsuka T, Kimura MT (2013a) Diversity and host associations of parasitoids attacking mycophagous drosophilids 
(Diptera: Drosophilidae) in northern and central Japan. Entomol Sci 16:227-234

Kasuya N, Mitsui H, Ideo S et al (2013b) Ecological, morphological and molecular studies on Ganaspis individuals (Hymenoptera: Figitidae) attacking Drosophila suzukii (Diptera: Drosophilidae). Appl Entomol Zool 48:87-92. https://doi.org/10.1007/s1335 5-012-0156-0

Kenis M, Tonina L, Eschen R et al (2016) Non-crop plants used as hosts by Drosophila suzukii in Europe. J Pest Sci 89:735-748. https://doi.org/10.1007/s10340-016-0755-6

Knoll V, Ellenbroek T, Romeis J, Collatz J (2017) Seasonal and regional presence of hymenopteran parasitoids of Drosophila in Switzerland and their ability to parasitize the invasive Drosophila suzukii. Sci Rep 7:40697

Kuhlmann U, Schaffner U, Mason PG (2006) Selection of non-target species for host specificity testing. Environmental impact of invertebrates for biological control of arthropods: methods and risk assessment. CABI, Wallingford, pp 15-37

Lee JC, Dreves AJ, Cave AM et al (2015) Infestation of wild and ornamental noncrop fruits by Drosophila suzukii (Diptera: Drosophilidae). Ann Entomol Soc Am 108:117-129. https://doi.org/10.1093/ aesa/sau014

Letunic I, Bork P (2016) Interactive tree of life (iTOL) v3: an online tool for the display and annotation of phylogenetic and other trees. Nucleic Acids Res 44:W242-245

Mason PG, Kabaluk JT, Spencer B, Gillespie DR (2013) Regulation of biological control in Canada. In: Mason PG, Gillespie DR (eds) Biological control programmes in Canada 2001-2012. CABI Publishing, Wallingford, pp 1-5

Matsuura A, Mitsui H, Kimura MT (2017) A preliminary study on distributions and oviposition sites of Drosophila suzukii (Diptera: Drosophilidae) and its parasitoids on wild cherry tree in Tokyo, central Japan. Appl Entomol Zool 52:429-437

Mitsui H, Kimura MT (2010) Distribution, abundance and host association of two parasitoid species attacking frugivorous drosophilid larvae in central Japan. Eur J Entomol 107:535-540

NCBI Taxonomy. https://www.ncbi.nlm.nih.gov/taxonomy

Nomano FY, Mitsui H, Kimura MT (2015) Capacity of Japanese Asobara species (Hymenoptera; Braconidae) to parasitize a fruit pest Drosophila suzukii (Diptera; Drosophilidae). J Appl Entomol 139:105-113

Nomano FY, Kasuya N, Matsuura A et al (2017) Genetic differentiation of Ganaspis brasiliensis (Hymenoptera: Figitidae) from East and Southeast Asia. Appl Entomol Zool 52:429-437

Novković B, Mitsui H, Suwito A, Kimura MT (2011) Taxonomy and phylogeny of Leptopilina species (Hymenoptera: Cynipoidea: Figitidae) attacking frugivorous drosophilid flies in Japan, with description of three new species. Entomol Sci 14:333-346
Pimentel D, McNair S, Janecka J et al (2001) Economic and environmental threats of alien plant, animal, and microbe invasions. Agric Ecosyst Environ 84:1-20

Poyet M, Havard S, Prevost G et al (2013) Resistance of Drosophila suzukii to the larval parasitoids Leptopilina heterotoma and Asobara japonica is related to haemocyte load. Physiol Entomol 38:45-53

R Core Team (2017) R: a language and environment for statistical. R Found Stat Comput Vienna, Austria

Roques A, Rabitsch W, Rasplus J-Y et al (2009) Alien terrestrial invertebrates of Europe. Handbook of alien species in Europe. Springer, Dordrecht, pp 63-79

Rossi-Stacconi MV, Buffington M, Daane KM et al (2015) Host stage preference, efficacy and fecundity of parasitoids attacking Drosophila suzukii in newly invaded areas. Biol Control 84:28-35

Rossi-Stacconi MV, Panel A, Baser N et al (2017) Comparative life history traits of indigenous. Biol Control 112:20-27

Urbaneja A, Llácer E, Tomás Ó et al (2000) Indigenous natural enemies associated with Phyllocnistis citrella (Lepidoptera: Gracillariidae) in Eastern Spain. Biol Control 18:199-207

van Alphen JJM, Janssen ARM (1981) Host selection by Asobara tabida Nees (Braconidae; Alysiinae) a larval parasitoid of fruit inhabiting Drosophila species. Netherlands J Zool 32:194-214

van Driesche RG, Hoddle MS (2016) Non-target effects of insect biocontrol agents and trends in host specificity since 1985 . CAB Rev 11:1-66

van Driesche RG, Murray TJ (2004) Overview of testing schemes and designs used to estimate host ranges. USDA Forest Service, Morgantown

van Lenteren JC, Bale J, Bigler F et al (2006) Assessing risks of releasing exotic biological control agents of arthropod pests. Annu Rev Entomol 51:609-634. https://doi.org/10.1146/annur ev.ento.51.110104.151129

Vazquez RJ, Porter SD, Briano JA (2004) Host specificity of a biotype of the fire ant decapitating fly Pseudacteon curvatus (Diptera: Phoridae) from northern Argentina. Environ Entomol $33: 1436-1441$

Wachi N, Nomano FY, Mitsui $\mathrm{H}$ et al (2015) Taxonomy and evolution of putative thelytokous species of Leptopilina (Hymenoptera: Figitidae) from Japan, with description of two new species. Entomol Sci 18:41-54

Walsh DB, Bolda MP, Goodhue RE et al (2011) Drosophila suzukii (Diptera: Drosophilidae): invasive pest of ripening soft fruit expanding its geographic range and damage potential. J Integr Pest Manag 2:1-8 